\title{
Existence and $\delta$-Approximate solution of implicit fractional pantograph equations in the frame of Hilfer-Katugampola operator
}

\author{
Mohammed A. Almalahi $a, b, * \infty{ }^{a}$, Satish K. Panchal ${ }^{a}$ \\ a Department of Mathematics, Dr.Babasaheb Ambedkar Marathwada University, \\ Aurangabad, (M.S),431001, India. \\ $\mathrm{b}$ Department of Mathematics, Hajjah University, Hajjah, Yemen.
}

\author{
• Received: 15.12.2020 • Accepted: 16.03.2021 • Published Online: 29.06.2021
}

\begin{abstract}
The major goal of this research paper is to investigate the existence and uniqueness of an implicit fractional pantograph equation in the frame of the Hilfer-Katugampola operator on the finite interval $[a, b]$ with mixed nonlocal conditions. Our analysis of the existence and uniqueness of solutions depends on some fixed point theorems such as Banach and Krasnoselskii. Moreover, we discuss the dependence of solutions on mixed nonlocal conditions by means of $\delta$-approximated solution. As an application, we provide an example to illustrate the validity of our results.
\end{abstract}

Keywords: Hilfer-Katugampola fractional derivative, Existence, Nonlocal conditions, $\delta$-approximate solution.

2010 MSC: 26A33, 34B10, 33E12, 49K40.

\section{Introduction}

In the last three decades, the study of fractional calculus has very caught importance and popularity among researchers due to their applicability in modeling many phenomena in the real-world such as propagation in complex medium, polymers, biological tissues, expansion of universe, earth sediments, etc. For more details, we refer the reader to the monographs $[1,2,3,4]$, and references therein. In order to meet the need of modeling more real-world problems in different fields of science and engineering, some researchers have realized necessary finding new fractional derivatives (FD). In this work, we consider Hilfer-Katugampola type fractional derivative which interpolates some fractional derivatives such as, Hilfer, Hilfer-Hadamard, Riemann-Liouville, Hadamard, Caputo, CaputoHadamard, see $[4,5,6,7,8]$ and the references therein. On the other hand, some researchers via different types of fractional derivatives studied the existence and stability of 
Ulam-Hyers, which can be found in $[10,11,12,13,14,15,16,17,18]$. A pantograph is an important tool employed in electric trains in order to collect electric currents from the overload lines. The pantograph equations have been modeled by Ockendon and Tayler in [19]. Persuaded by the importance of these equations, many researchers have generalized these equations into various kinds and presented the solvability theoretically and numerically portion of such problems. For additional details, see [20, 21, 22, 23, 24, 25, 26] and the references therein. Recently, Abdo et al. [27] Considered the pantograph (FDEs)

$$
\left\{\begin{array}{c}
{ }^{A B C} \mathbf{D}_{a^{+}} \vartheta(\sigma)=f(\sigma, \vartheta(\sigma), \vartheta(\mu(\sigma))), \mu \in(0,1) \\
\vartheta(a)=\sum_{j=1}^{n} \tau_{j} \vartheta\left(\kappa_{j}\right), k_{j} \in(a, T)
\end{array}\right.
$$

The authors by means of Banach's contraction principle and Krasnoselskii fixed point theorem studied the existence and uniqueness results and by using Gronwall's inequality in the frame of $(A B C)$ discussed the Ulam-Hyers stabilities. Asawasamrit et. al. [28] by means of some fixed point theorems studied existence, uniqueness and different type of Ulam stability results of the following problem

$$
\left\{\begin{array}{c}
D^{q} \vartheta(\sigma)=f(\sigma, \vartheta(\sigma)), 0<q<1, \sigma \in J:=[0, T] \\
\sum_{i=1}^{m} \gamma_{i} \vartheta\left(\eta_{i}\right)+\sum_{j=1}^{n} \tau_{j} D_{0^{+}}^{\lambda_{j}} \vartheta\left(\kappa_{j}\right)+\sum_{i=1}^{m} \theta_{i} \mathcal{J}_{0^{+}}^{\psi_{i}} \vartheta\left(\varpi_{i}\right)=A \in \mathbb{R},
\end{array}\right.
$$

where $\mathrm{D}^{\mathrm{q}}, \mathrm{D}^{\lambda_{j}}$ are the Caputo (FD) of order $\mathrm{q}$ and $\lambda_{j}$, respectively, $0<\lambda_{j}<\mathrm{q} \leqslant 1$, $j=1,2, \ldots, n, \gamma_{i}, \tau_{j}, \theta_{r} \in \mathbb{R}$ and $\eta_{i}, \kappa_{j}, \varpi_{i} \in J$.

On the other hand, Almalahi et. al. [29] via some properties of Mittag-Leffler functions and some fixed point theorems studied the existence, uniqueness and different type of Ulam stability results of the following Hilfer-Katugampola (FDEs)

$$
\left\{\begin{array}{l}
\rho{ }^{\rho} \mathrm{D}_{0^{+}, \beta}^{\mathrm{q}} \vartheta(\sigma)=\lambda \vartheta(\sigma)+\mathrm{f}(\sigma, \vartheta(\sigma)), \lambda<0,0<\alpha<1,0 \leqslant \beta \leqslant 1, \sigma \in J:=(0, \mathrm{~T}] \\
{ }^{\rho} \mathcal{J}_{0^{+}}^{1-\gamma} \vartheta\left(0^{+}\right)=\sum_{i=1}^{m} \delta_{i} \rho \mathcal{J}_{0^{+}}^{\eta} \vartheta\left(\tau_{i}\right), q \leqslant \gamma=\mathrm{q}+\beta-\mathrm{q} \beta<1 \quad \tau_{i} \in(a, T],
\end{array}\right.
$$

where ${ }^{\rho} D_{a^{+}}^{q, \beta}(\cdot)$ denotes the Hilfer-Katugampola (FD) of order $q \in(0,1)$ and type $\beta \in$ $[0,1]$ and ${ }^{\rho} \mathrm{J}_{0^{+}}^{1-\gamma}$ is a generalized fractional integral (GFI) of order $1-\gamma, \rho>0$. Here $f: J \times \mathbb{R} \longrightarrow \mathbb{R}$ is a continuous function, $\tau_{\mathfrak{i}}(i=0,1,2, \ldots ., \mathrm{m})$ are prefixed points satisfying $0<\tau_{1} \leqslant \tau_{2} \leqslant \ldots . \leqslant \tau_{m}<\mathrm{T}$, and $\lambda<0, \delta_{i} \in \mathbb{R}$.

Motivated by the above argumentations, the intent of this work is to investigate the existence and uniqueness of solutions as well as the dependence of solutions by means of $\delta$-approximated solution of the implicit fractional pantograph equation:

$$
\left\{\begin{array}{c}
\rho D_{a^{+}}^{q, \beta} \vartheta(\sigma)=f\left(\sigma, \vartheta(\sigma), \vartheta(\mu(\sigma)),{ }^{\rho} D_{a^{+}}^{q, \beta} \vartheta(\sigma)\right), \mu \in(0,1), \sigma \in J:=[a, T] \\
\sum_{i=1}^{m} \theta_{i} \rho \mathcal{J}_{a^{+}}^{\psi_{i}} \vartheta\left(\varpi_{i}\right)=B-\sum_{j=1}^{n} \tau_{j}{ }^{\rho} D_{a^{+}}^{\lambda_{j}, \beta} \vartheta\left(k_{j}\right) \in \mathbb{R} .
\end{array}\right.
$$

where ${ }^{\rho} \mathrm{D}_{\mathrm{a}^{+}}^{\mathrm{q}, \beta}(\cdot),{ }^{\rho} \mathrm{D}_{\mathrm{a}^{+}}^{\lambda_{j}, \beta}(\cdot)$ are the Hilfer-Katugampola (FD) of order $\mathrm{q}$ and $\lambda_{j}$ respectively, $q \in(0,1)$ and type $\beta \in[0,1], q \geqslant \lambda_{j}+\beta\left(1-\lambda_{j}\right),(j=0,1,2, \ldots, n),{ }^{\rho} \mathcal{J}_{a^{+}}^{\psi_{i}}, \rho_{\mathcal{J}} a_{a^{+}}$are the generalized fractional integral of order $\psi_{i}, q,(i=0,1,2, \ldots ., m)$ respectively, $\theta_{i}, \tau_{j} \in \mathbb{R}-\{0\}$ and $\varpi_{i}, \kappa_{j} \in J$ are prefixed points. $f: J \times \mathbb{R}^{3} \longrightarrow \mathbb{R}$ is continuous function. We confirm that our proposed problem (1.1) is a fractional integral multi-order and fractional derivative multi-order problem, which means our results yield some results related to choosing 
the parameters. In addition, we note that, if $\theta_{i}=0$ then (1.1) reduce to implicit fractional pantograph equations subjected to fractional derivative multi-order conditions, if $\tau_{j}=0$ then (1.1) reduce to implicit fractional pantograph equations subjected to fractional integral multi-order conditions. We investigate the existence and uniqueness of solution as well as dependence of solutions and uniqueness by means of $\delta$-approximated solution of the proposed problem. The fractional derivative ${ }^{\rho} \mathrm{D}_{\mathrm{a}^{+}}^{q, \beta}$ is an interpolator some fractional derivatives such as Hilfer (if $\rho=1$ ), Hilfer-Hadamard (if $\rho=0^{+}$), generalized (if $\beta=0$ ), Caputo-type (if $\beta=1$ ), Riemann-Liouville (if $\beta=0, \rho=1$ ), Hadamard (if $\beta=0$, $\rho=0^{+}$), Caputo (if $\beta=0, \rho=1$ ), Caputo-Hadamard (if $\beta=1, \rho=0^{+}$), Liouville (if $\beta=0, \rho=1, a=0$ ) and Weyl (if $\beta=0, \rho=1, a=-\infty$ ). To the best of our knowledge, this is the first work dealing with the implicit fractional pantograph equations in the frame of Hilfer-Katugampola operator. In consequence, the results of this work will be a useful contribution to the existing literature on this topic.

This paper is organized as follows. In Section 2, we present notations, auxiliary lemmas and some basic definitions which are used throughout the paper. In Section 3, we discuss the existence and uniqueness results for a system 1.1. In Section 4, we discuss dependence and uniqueness of solutions by means of $\delta$-approximate solution. In section 5 , we provide some examples to illustrate the validity of our results. Concluding remarks about our results in the last section.

\section{Background material and auxiliary results}

In order to achieve our main purposes, we present here some definitions and basic auxiliary results that are required throughout our paper. Let $0<a<T, J:=[a, T]$ and let $\mathrm{C}(\mathrm{J})$ be the Banach space of all continuous function from $\mathrm{J}$ into $\mathbb{R}$ with supremum norm $\|\vartheta\|_{\infty}=\sup \{|\vartheta(\sigma)|: \sigma \in J\}$. Let $X_{c}^{p}(J),(c \in \mathbb{R}, 1 \leqslant P \leqslant \infty)$ be the space of the complex Lebesgue measurable functions $\vartheta$ on J for which $\|\vartheta\|_{X_{c}^{p}}<\infty$, where

$$
\|\vartheta\|_{X_{c}^{p}}=\left(\int_{a}^{T}\left|\sigma^{c} \vartheta(\sigma)\right|^{p} \frac{d \sigma}{\sigma}\right)^{\frac{1}{p}} .
$$

In the case, $c=\frac{1}{p}$, the space $X_{\frac{1}{p}}^{p}(J)=L_{p}(J)$.

Definition 2.1. [30] Let $q \in \mathbb{R}_{+}, c \in \mathbb{R}$ and $\vartheta(\sigma) \in X_{c}^{p}(J)$. Then, the generalized leftsided fractional integral ${ }^{\rho \mathcal{J}_{\mathbf{a}^{+}}^{\mathrm{q}}}$ of order $\mathrm{q}>0$ is defined by

$$
\rho_{\mathcal{J}_{a^{+}}}^{q} \vartheta(\sigma)=\frac{1}{\Gamma(q)} \int_{a}^{\sigma}\left(\frac{\sigma^{\rho}-s^{\rho}}{\rho}\right)^{q-1} s^{\rho-1} \vartheta(s) d s, \quad \sigma>a, \rho>0 .
$$

Definition 2.2. [30] The Katugampola (FD) of order $q \in \mathbb{R}_{+} \backslash \mathbb{N}, \rho>0$ is defined by:

$$
{ }^{\rho} D_{a^{+}}^{q} \vartheta(\sigma)=\frac{1}{\Gamma(n-q)}\left(\sigma^{1-\rho} \frac{d}{d \sigma}\right)^{n} \int_{a}^{T} s^{\rho-1}\left(\frac{\sigma^{\rho}-s^{\rho}}{\rho}\right)^{n-q+1} \vartheta(s) d s,
$$

where $n=[q]+1$. 
Definition 2.3. [31] Let $n-1<q<n$ and $0 \leqslant \beta \leqslant 1$. Then, the Hilfer-Katugampola (FD) with respect to $\sigma$, with $\rho>0$ of a function $\vartheta$ is defined by:

$$
{ }^{\rho} D_{a^{+}}^{q, \beta} \vartheta(\sigma)=\rho_{\mathcal{J}}^{\beta(n-q)}\left(\sigma^{1-\rho} \frac{d}{d \sigma}\right)^{n} \rho_{\mathcal{J}^{+}}^{(1-\beta)(n-q)} \vartheta(\sigma),
$$

the operator ${ }^{\rho} \mathrm{D}_{\mathrm{a}^{+}}^{\mathrm{q}, \beta}$ can be expressed by

$$
\begin{aligned}
\rho^{\rho} D_{a^{+}}^{q, \beta} & =\rho_{\mathcal{J}_{a^{+}}^{\beta(n-q)}}\left(\sigma^{1-\rho} \frac{d}{d \sigma}\right)^{n} \rho_{\mathcal{J}_{a^{+}}^{n-\gamma}} \\
& =\rho_{\mathcal{J}_{a^{+}}^{\beta(n-q)}} \rho D_{a^{+}}^{\gamma}, \gamma=q+n \beta-q \beta,
\end{aligned}
$$

In this paper, due to $\mathrm{q} \in(0,1)$, we consider $\mathrm{n}=1$.

Lemma 2.4. [31] Let $\rho^{\rho} \mathcal{J}_{\mathrm{a}^{+}}^{\mathrm{q}}$ and ${ }^{\rho} \mathrm{D}_{\mathrm{a}^{+}}^{\mathrm{q}}$ are generalized left-sided fractional integral and derivative respectively defined in (2.1) and (2.2). Then, for $\mathrm{q}>0$ and $\beta>0$, we have

$$
\begin{aligned}
& \left(\rho_{\mathcal{J}_{a^{+}}^{q}}\left(\frac{\sigma^{q}-a^{\rho}}{\rho}\right)^{\beta-1}\right)(\sigma)=\frac{\Gamma(\beta)}{\Gamma(\beta+q)}\left(\frac{\sigma^{q}-a^{\rho}}{\rho}\right)^{q+\beta-1}, q \geqslant 0, \beta>0, \\
& \left({ }^{\rho} D_{a^{+}}^{q}\left(\frac{\sigma^{q}-a^{\rho}}{\rho}\right)^{q-1}\right)(\sigma)=0,0<q<1 .
\end{aligned}
$$

Lemma 2.5. Let $\gamma=\mathrm{q}+\beta-\mathrm{q} \beta$ where $\mathrm{q} \in(0,1)$ and $\beta \in[0,1]$. If $\vartheta \in \mathrm{C}_{\gamma, \rho}^{\gamma}(\mathrm{J})$, then

$$
\begin{aligned}
& \rho_{\mathcal{J}_{\mathbf{T}^{+}}^{\gamma}}^{\gamma}{ }^{\rho} \mathrm{D}_{\mathbf{a}^{+}}^{\gamma} \vartheta=\rho_{\mathcal{J}_{\mathbf{a}^{+}}^{q}}{ }^{\rho} \mathrm{D}_{\mathbf{a}^{+}}^{\mathrm{q}, \beta} \vartheta, \\
& { }^{\rho} \mathrm{D}_{\mathrm{a}^{+}}^{\gamma}{ }^{\rho} \mathcal{J}_{\mathrm{a}^{+}}^{\mathrm{q} \vartheta}={ }^{\rho} \mathrm{D}_{\mathrm{a}^{+}}^{\beta(1-\mathrm{q})} \vartheta \text {. }
\end{aligned}
$$

Lemma 2.6. [31] Let $\mathrm{q} \in(0,1)$. Then for $\sigma \in \mathrm{J}$, we have

$$
\rho_{\mathcal{J}_{a^{+}}}^{q}{ }^{\rho} D_{a^{+}}^{q} \vartheta(\sigma)=\vartheta(\sigma)-\frac{\rho \mathcal{J}_{a^{+}}^{1-\gamma} \vartheta(a)}{\Gamma(\gamma)}\left(\frac{\sigma^{q}-a^{\rho}}{\rho}\right)^{\gamma-1} .
$$

Theorem 2.7. [32] Let $\mathrm{X}$ be a Banach space and $\mathrm{K} \subset \mathrm{X}$ be a nonempty, closed, convex and bounded. If there is tow operators $\Phi^{1}, \Phi^{2}$ such that for all $u, v \in X$, imply $\Phi^{1} u+\Phi^{2} v \in X$ also $\Phi^{1}$ is compact and continuous and $\Phi^{2}$ is a contraction mapping, then there exists a function $z \in \mathrm{K}$ such that $z=\Phi^{1} z+\Phi^{2} z$.

Lemma 2.8. Let $\gamma=q+\beta-q \beta, q \in(0,1)$ and $\beta \in[0,1]$. If $q \geqslant \lambda_{j}+\beta\left(1-\lambda_{j}\right)$ and $\vartheta \in \mathrm{C}(\mathrm{J})$, then

$$
\rho^{\rho} \mathrm{D}_{\mathrm{a}^{+}}^{\lambda, \beta \rho} \rho_{\mathcal{J}^{+}}^{q} \vartheta(\sigma)=\rho_{\mathcal{J}^{+}}^{q-\lambda} \vartheta(\sigma) .
$$

Proof. From definition 2.3, $\mathrm{n}=1$, we have

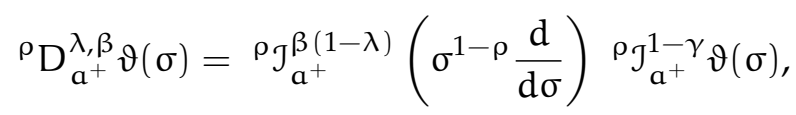


where $\gamma=\lambda+\beta-\lambda \beta$. Then, we have

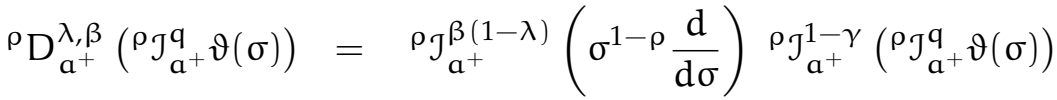

$$
\begin{aligned}
& =\rho_{\mathcal{J}_{\mathbf{a}^{+}} \beta(1-\lambda)}\left(\sigma^{1-\rho} \frac{d}{d \sigma}\right) \rho_{\mathcal{J}_{\mathbf{a}^{+}}^{1-\gamma+q}} \vartheta(\sigma) \\
& =\rho_{\mathcal{J}} \beta(1-\lambda)\left(\sigma^{1-\rho} \frac{d}{d \sigma}\right) \\
& \frac{1}{\Gamma(1-\gamma+q)} \int_{a}^{\sigma}\left(\frac{\sigma^{\rho}-s^{\rho}}{\rho}\right)^{q-\gamma} s^{\rho-1} \vartheta(s) d s \\
& =\rho_{\mathcal{J}^{\prime}}^{\beta(1-\lambda)} \frac{1}{\Gamma(q-\gamma)} \int_{a}^{\sigma}\left(\frac{\sigma^{\rho}-s^{\rho}}{\rho}\right)^{q-\gamma-1} s^{\rho-1} \vartheta(s) d s \\
& =\rho_{\mathcal{J}_{\mathbf{a}^{+}}^{\mathrm{q}-\lambda}} \vartheta(\sigma) \text {. }
\end{aligned}
$$

Theorem 2.9. Let $\gamma=\mathrm{q}+\beta-\mathrm{q} \beta, \mathrm{q} \in(0,1), \beta \in[0,1]$ and $\mathrm{h}: \mathrm{J} \longrightarrow \mathbb{R}$ be a continuous function such that $\mathrm{h} \in \mathrm{C}(\mathrm{J})$. A function $\vartheta \in \mathrm{C}^{\gamma}(\mathrm{J})$ is a solution of problem

$$
\left\{\begin{array}{c}
\rho \mathrm{D}_{\mathrm{a}^{+}}^{\mathrm{q}, \beta} \vartheta(\sigma)=h(\sigma), 0<\mathrm{q}<1,0 \leqslant \beta \leqslant 1, \sigma \in J \\
\sum_{i=1}^{m} \theta_{i} \rho_{\mathfrak{j}^{+}}^{\psi_{i}} \vartheta\left(\varpi_{i}\right)=B-\sum_{j=1}^{n} \tau_{j}{ }^{\rho} \mathrm{D}_{\mathbf{a}^{+}}^{\lambda_{j}, \beta} \vartheta\left(\kappa_{j}\right), B \in \mathbb{R} .
\end{array}\right.
$$

if and only if $\vartheta$ satisfies the following mixed type integral

$$
\begin{aligned}
\vartheta(\sigma)= & \frac{\left(\frac{\sigma^{\rho}-a^{\rho}}{\rho}\right)^{\gamma-1}}{\Psi \Gamma(\gamma)}\left(B-\sum_{i=1}^{m} \theta_{i} \rho_{\mathcal{J}_{a^{+}}^{q}}^{q+\psi_{i}} h(s)\left(\varpi_{i}\right)-\sum_{j=1}^{n} \tau_{j} \rho_{\mathcal{J}_{a^{+}}^{q-\lambda_{j}}} h(s)\left(\kappa_{j}\right)\right) \\
& +\rho_{\mathcal{J}_{\mathbf{a}^{+}}^{q}}^{q} h(s)(\sigma),
\end{aligned}
$$

where

$$
\Psi=\sum_{i=1}^{m} \frac{\theta_{i}}{\Gamma\left(\gamma+\psi_{i}\right)}\left(\frac{\varpi_{i}^{\rho}-a^{\rho}}{\rho}\right)^{\gamma+\psi_{i}-1}+\sum_{j=1}^{n} \frac{\tau_{j}}{\Gamma\left(\gamma-\lambda_{j}\right)}\left(\frac{\kappa_{j}^{\rho}-a^{\rho}}{\rho}\right)^{\gamma-\lambda_{j}-1} \neq 0
$$

Proof. In view of (2.3), first equation of (2.4) can be written as

$$
\rho_{\mathcal{J}^{\prime}}^{\beta(1-q) \rho} \rho_{\mathbf{a}^{+}}^{\gamma} \mathrm{a}_{\mathbf{a}^{+}}^{\gamma} \vartheta(\sigma)=h(\sigma)
$$

Applying the operator ${ }^{\rho} \mathcal{J}_{a^{+}}^{q}$ to both sides of (2.7) and using Lemma 2.6, we get

$$
\vartheta(\sigma)=\frac{\rho \mathcal{J}_{\mathbf{a}^{+}}^{1-\gamma} \vartheta(\mathbf{a})}{\Gamma(\gamma)}\left(\frac{\sigma^{q}-a^{\rho}}{\rho}\right)^{\gamma-1}+\rho_{\mathcal{J}_{\mathbf{a}^{+}}^{q}} h(s)(\sigma)
$$

Denoting $c=\rho \mathcal{J}_{\mathbf{a}^{+}}^{1-\gamma} \vartheta(a)$ in (2.8) yields

$$
\vartheta(\sigma)=\frac{c}{\Gamma(\gamma)}\left(\frac{\sigma^{\rho}-a^{\rho}}{\rho}\right)^{\gamma-1}+\rho_{\mathcal{J}_{a^{+}}^{q}} h(s)(\sigma) .
$$


Taking the operator $\rho \mathcal{J}_{\mathbf{a}^{+}}^{\psi_{i}}$ into both sides of (2.9) with replace $\sigma$ by $\varpi_{i}$ and using lemma 2.4, we get

$$
\rho_{\mathcal{J}_{a^{+}}}^{\psi_{i}} \vartheta\left(\varpi_{i}\right)=\frac{c}{\Gamma\left(\gamma+\psi_{i}\right)}\left(\frac{\varpi_{i}^{\rho}-a^{\rho}}{\rho}\right)^{\gamma+\psi_{i}-1}+\rho_{\mathcal{J}_{a^{+}}^{q+}+\psi_{i}} h(s)\left(\varpi_{\mathfrak{i}}\right) .
$$

Taking again the operator ${ }^{\rho} \mathrm{D}_{\mathrm{a}^{+}}^{\lambda_{j}, \beta}$ into both sides of (2.9) with replace $\sigma$ by $\kappa_{j}$ and using lemmas 2.4, 2.8, we obtain

$$
{ }^{\rho} D_{a^{+}}^{\lambda_{j}, \beta} \vartheta\left(\kappa_{j}\right)=\frac{c}{\Gamma\left(\gamma-\lambda_{j}\right)}\left(\frac{\kappa_{j}^{\rho}-a^{\rho}}{\rho}\right)^{\gamma-\lambda_{j}-1}+\rho_{\mathcal{J}_{a^{+}}^{q-\lambda_{j}}} h(s)\left(\kappa_{j}\right) .
$$

Thus

$$
\begin{aligned}
& \sum_{i=1}^{m} \theta_{i} \rho_{\mathcal{J}^{+}}^{\psi_{i}} \vartheta\left(\varpi_{i}\right)+\sum_{j=1}^{n} \tau_{j}{ }^{\rho} D_{a^{+}}^{\lambda_{j}, \beta} \vartheta\left(\kappa_{j}\right) \\
& =\sum_{i=1}^{m} \frac{\theta_{i} c}{\Gamma\left(\gamma+\psi_{i}\right)}\left(\frac{\varpi_{i}^{\rho}-a^{\rho}}{\rho}\right)^{\gamma+\psi_{i}-1}+\sum_{i=1}^{m} \theta_{i} \rho_{\mathcal{J}_{a^{+}}^{q+}}{ }^{i} h(s)\left(\varpi_{i}\right) \\
& +\sum_{j=1}^{n} \frac{\tau_{j} c}{\Gamma\left(\gamma-\lambda_{j}\right)}\left(\frac{\kappa_{j}^{\rho}-a^{\rho}}{\rho}\right)^{\gamma-\lambda_{j}-1}+\sum_{j=1}^{n} \tau_{j}{ }^{\rho} \mathcal{J}_{a^{+}}^{q-\lambda_{j}} h(s)\left(\kappa_{j}\right) \\
& =c\left(\sum_{i=1}^{m} \frac{\theta_{i}}{\Gamma\left(\gamma+\psi_{i}\right)}\left(\frac{\varpi_{i}^{\rho}-a^{\rho}}{\rho}\right)^{\gamma+\psi_{i}-1}+\sum_{j=1}^{n} \frac{\tau_{j}}{\Gamma\left(\gamma-\lambda_{j}\right)}\left(\frac{\kappa_{j}^{\rho}-a^{\rho}}{\rho}\right)^{\gamma-\lambda_{j}-1}\right)
\end{aligned}
$$

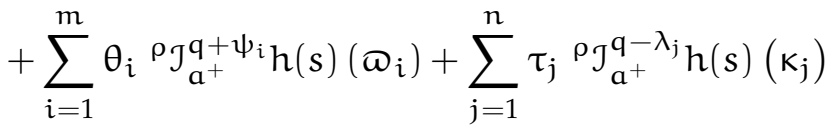

It follows from the condition $\left(\sum_{i=1}^{m} \theta_{i} \rho I_{a^{+}}^{\psi_{i}} \vartheta\left(\varpi_{i}\right)=B-\sum_{j=1}^{n} \tau_{j}{ }^{\rho} D_{a^{+}}^{\lambda_{j}, \beta} \vartheta\left(\kappa_{j}\right)\right)$ that

$$
c=\frac{1}{\Psi}\left(B-\sum_{i=1}^{m} \theta_{i} \rho_{\mathcal{J}_{a^{+}}}^{q+\psi_{i}} h(s)\left(\varpi_{i}\right)-\sum_{j=1}^{n} \tau_{j} \rho_{\mathcal{J}_{a^{+}}^{q-\lambda}}^{q-\lambda_{j}} h(s)\left(\kappa_{j}\right)\right) .
$$

Substitute (2.12) into (2.9), we get (2.5). Conversely, let $\vartheta \in \mathrm{C}^{\gamma}(\mathrm{J})$ satisfying (2.5). We need to show that $\vartheta$ also satisfies (2.4). For that, applying the operator ${ }^{\rho} \mathrm{D}_{\mathrm{a}^{+}}^{\gamma}$ on both sides of (2.5). Then, from Lemmas 2.4 and 2.5, we get

$$
{ }^{\rho} \mathrm{D}_{\mathrm{a}^{+}}^{\gamma} \vartheta(\sigma)={ }^{\rho} \mathrm{D}_{\mathrm{a}^{+}}^{\beta(1-\mathrm{q})} \mathrm{h}(\sigma)
$$

By the definition of $\mathrm{C}^{\gamma}(\mathrm{J})$ and $\vartheta \in \mathrm{C}^{\gamma}(\mathrm{J})$, we have ${ }^{\rho} \mathrm{D}_{\mathrm{a}^{+}}^{\gamma} \vartheta \in \mathrm{C}(\mathrm{J})$. Consequently, ${ }^{\rho} \mathrm{D}_{\mathrm{a}^{+}}^{\beta(1-\mathrm{q})} \vartheta={ }^{\rho} \mathrm{D}^{1 \rho} \mathrm{I}_{\mathrm{a}^{+}}^{1-\beta(1-\mathrm{q})} \vartheta \in \mathrm{C}(\mathrm{J})$. For any $\vartheta \in \mathrm{C}(\mathrm{J}), \sigma \in \mathrm{J}$, it is obvious that ${ }^{\rho} \mathrm{I}_{\mathrm{a}^{+}}^{1-\beta(1-q)} \vartheta \in \mathrm{C}^{1}(J)$. Now, multiplying both sides of (2.13) by ${ }^{\rho} \mathrm{I}_{\mathrm{a}^{+}}^{\beta(1-q)}$, using lemma 2.6, we can write

$$
{ }^{\rho} D_{a^{+}}^{q, \beta} \vartheta(\sigma)=h(\sigma)-\frac{\rho I_{a^{+}}^{1-\beta(1-q)} h(a)}{\Gamma(\beta(1-q))}\left(\frac{\sigma^{q}-a^{\rho}}{\rho}\right)^{\beta(1-q)-1},
$$


where ${ }^{\rho} \mathrm{I}_{\mathrm{a}^{+}}^{1-\beta(1-\mathrm{q})} \mathrm{h}(\mathrm{a})=0$. Hence (2.14) reduces to

$$
{ }^{\rho} \mathrm{D}_{\mathrm{a}^{+}}^{\mathrm{q}, \beta} \vartheta(\sigma)=h(\sigma)
$$

On the other hand, applying the operator ${ }^{\rho} \mathcal{J}_{a^{+}}^{\psi_{i}}$ and ${ }^{\rho} D_{a^{+}}^{\lambda_{j}, \beta}$ to both sides of (2.5) with using Lemmas 2.8 and 2.4 , for $i=1,2, \ldots ., \mathrm{m}$, and $j=1,2, \ldots . n$, we obtain

$$
\begin{aligned}
& \sum_{i=1}^{m} \theta_{i} \rho_{\mathcal{J}}^{\psi_{a^{+}}} \vartheta\left(\varpi_{i}\right)=\sum_{i=1}^{m} \theta_{i} \frac{\frac{1}{\Gamma\left(\psi_{i}+\gamma\right)}\left(\frac{\Phi_{i}^{\rho}-a^{\rho}}{\rho}\right)^{\psi_{i}+\gamma-1}}{\Psi} \\
& \left(B-\sum_{i=1}^{m} \theta_{i} \rho_{\mathcal{J}_{a^{+}}^{q+}+\psi_{i}} h(s)\left(\varpi_{i}\right)-\sum_{j=1}^{n} \tau_{j} \rho_{\mathcal{J}_{a^{+}}^{q-} \lambda_{j}} h(s)\left(\kappa_{j}\right)\right) \\
& +\sum_{i=1}^{m} \theta_{i} \rho_{\mathcal{J}_{a^{+}}^{q+} \psi_{i}} h(s)\left(\varpi_{i}\right), \\
& \sum_{j=1}^{n} \tau_{j}^{\rho} D_{a^{+}}^{\lambda_{j}, \beta} \vartheta\left(\kappa_{j}\right)=\sum_{j=1}^{n} \tau_{j} \frac{\frac{1}{\Gamma\left(\gamma-\lambda_{j}\right)}\left(\frac{\kappa_{j}^{\rho}-a^{\rho}}{\rho}\right)^{\gamma-\lambda_{j}-1}}{\Psi} \\
& \left(B-\sum_{i=1}^{m} \theta_{i} \rho_{\mathcal{J}_{a^{+}}^{q+\psi_{i}}} h(s)\left(\varpi_{i}\right)-\sum_{j=1}^{n} \tau_{j}{ }^{\rho_{\mathcal{J}}}{ }_{a^{+}}^{q-\lambda_{j}} h(s)\left(\kappa_{j}\right)\right) \\
& +\sum_{j=1}^{n} \tau_{j}{ }^{\rho} \rho_{a^{+}}^{q-\lambda_{j}} h(s)\left(\kappa_{j}\right),
\end{aligned}
$$

where $\Psi$ is given by (2.6). Therefore

$$
\sum_{i=1}^{m} \theta_{i} \rho \mathcal{J}_{a^{+}}^{\psi_{i}} \vartheta\left(\varpi_{i}\right)+\sum_{j=1}^{n} \tau_{j}{ }^{\rho} D_{a^{+}}^{\lambda_{j}, \beta} \vartheta\left(\kappa_{j}\right)=B .
$$

The lemma is proved.

\section{Exestence of solution}

In this part, we will prove our main results such as existence and uniqueness of solution for proposed problem. In order to obtain our results, we deliver the following assumptions

$\left(\mathrm{H}_{1}\right): f: J \times \mathbb{R} \times \mathbb{R} \times \mathbb{R} \rightarrow \mathbb{R}$ is a continuous function and there exist a constant $\mathfrak{R}_{\mathrm{f}}>0$ such that

$$
|f(\sigma, u, v, z)-f(\sigma, \bar{u}, \bar{v}, \bar{z})| \leqslant \Re_{\mathrm{f}}(|\mathrm{u}-\overline{\mathrm{u}}|+|v-\bar{v}|+|z-\bar{z}|),
$$

for any $u, v, z, \bar{u}, \bar{v}, \bar{z} \in \mathbb{R}$ and $\sigma \in \mathrm{J}$. 
$\left(\mathrm{H}_{2}\right)$ : The following inequality holds

$$
\begin{aligned}
\Delta= & \frac{2 \Re_{f}}{\left(1-\Re_{f}\right) \Psi \Gamma(\gamma)}\left(\sum_{i=1}^{m} \frac{\theta_{i}}{\Gamma\left(q+\psi_{i}+1\right)}\left(\frac{\varpi_{i}^{\rho}-a^{\rho}}{\rho}\right)^{q+\psi_{i}}\right. \\
& \left.+\sum_{j=1}^{n} \frac{\tau_{j}}{\Gamma\left(q-\lambda_{j}+1\right)}\left(\frac{\kappa_{j}^{\rho}-a^{\rho}}{\rho}\right)^{q-\lambda_{j}}\right) \\
& +\frac{2 \Re_{f}}{1-\Re_{f}} \frac{1}{\Gamma(q+1)}\left(\frac{T^{\rho}-a^{\rho}}{\rho}\right)^{q}<1
\end{aligned}
$$

In the light of Theorem 2.9, we have the following theorem.

Theorem 3.1. Let $\gamma=\mathrm{q}+\beta-\mathrm{q} \beta$ where $\mathrm{q} \in(0,1)$ and $\beta \in[0,1]$, let $\mathrm{F}: \mathrm{J} \times \mathbb{R} \times \mathbb{R} \times \mathbb{R} \longrightarrow$ $\mathbb{R}$ be a continuous function such that $\mathrm{F} \in \mathrm{C}(\mathrm{J})$ for all $\vartheta \in \mathrm{C}(\mathrm{J})$. A function $\vartheta \in \mathrm{C}^{\gamma}(\mathrm{J})$ is a solution of problem (1.1) if and only if $\vartheta$ is the fixed point (FP) of the operator $\mathcal{S}: \mathrm{C}(\mathrm{J}) \rightarrow$ $\mathrm{C}(\mathrm{J})$ defined as

$$
\begin{aligned}
\mathcal{S} \vartheta(\sigma)= & \frac{\left(\frac{\sigma^{\rho}-a^{\rho}}{\rho}\right)^{\gamma-1}}{\Psi \Gamma(\gamma)}\left(B-\sum_{i=1}^{m} \theta_{i} \rho_{\mathcal{J}}^{q+} a^{+} \psi_{i} F_{\vartheta}(s)\left(\varpi_{i}\right)\right. \\
& \left.-\sum_{j=1}^{n} \tau_{j} \rho_{\mathcal{J}_{a^{+}}^{q-}}^{q-\lambda_{j}} F_{\vartheta}(s)\left(\kappa_{j}\right)\right)+{ }^{\rho} \mathcal{J}_{a^{+}}^{q} F_{\vartheta}(s)(\sigma),
\end{aligned}
$$

where $F_{\vartheta}(s)=f\left(s, \vartheta(s), \vartheta(\mu(s)),{ }^{\rho} D_{a^{+}}^{q, \beta} \vartheta(s)\right)$.

Theorem 3.2. Assume that $\left(H_{1}\right)$ and $\left(H_{2}\right)$ holds, Then the problem (1.1) has a unique solution in $\mathrm{C}^{\gamma}(\mathrm{J})$.

Proof. Step (1): We will show that the operator $\mathcal{S}$ which defined in 3.2 has a unique fixed point $\vartheta \in \mathrm{C}(\mathrm{J})$. For that let $\vartheta, v \in \mathrm{C}(\mathrm{J}), \sigma \in \mathrm{J}$, then, we have

$$
\begin{aligned}
& \|\vartheta-v\|=\max _{\sigma \in J}|\mathcal{S} \vartheta(\sigma)-\mathcal{S} v(\sigma)| \\
\leqslant & \frac{\left(\frac{\sigma^{\rho}-a^{\rho}}{\rho}\right)^{\gamma-1}}{\Psi \Gamma(\gamma)}\left(\sum_{i=1}^{m} \theta_{i} \rho_{\mathcal{J}_{a^{+}}^{q+}+\psi_{i}}\left|F_{\vartheta}(s)-F_{v}(s)\right|\left(\varpi_{i}\right)\right. \\
& \left.+\sum_{j=1}^{n} \tau_{j} \rho_{\mathcal{J}_{a^{+}}^{q-\lambda_{j}}}^{q}\left|F_{\vartheta}(s)-F_{v}(s)\right|\left(\kappa_{j}\right)\right)+\rho_{\mathcal{J}_{a^{+}}^{q}}\left|F_{\vartheta}(s)-F_{v}(s)\right|(\sigma) \\
\leqslant & \frac{2 \Re_{f}}{\left(1-\Re_{f}\right) \Psi \Gamma(\gamma)}\left(\sum_{i=1}^{m} \theta_{i} \rho_{\mathcal{J}_{a^{+}}^{q+} \psi_{i}}|\vartheta(s)-v(s)|\left(\varpi_{i}\right)\right. \\
& \left.+\sum_{j=1}^{n} \tau_{j} \rho_{\mathcal{J}_{a^{+}}^{q-\lambda}}^{q-\lambda_{j}}|\vartheta(s)-v(s)|\left(\kappa_{j}\right)\right)+\frac{2 \Re_{f}}{1-\Re_{f}} \rho_{\mathcal{J}_{a^{+}}^{q}}^{q}|\vartheta(s)-v(s)|(\sigma)
\end{aligned}
$$




$$
\begin{aligned}
\leqslant & \frac{2 \mathfrak{R}_{f}}{\left(1-\mathfrak{R}_{\mathrm{f}}\right) \Psi \Gamma(\gamma)}\left(\sum_{i=1}^{m} \frac{\theta_{i}}{\Gamma\left(\mathrm{q}+\psi_{i}+1\right)}\left(\frac{\varpi_{i}^{\rho}-a^{\rho}}{\rho}\right)^{\mathrm{q}+\psi_{i}}\|\vartheta-v\|\right. \\
& \left.+\sum_{j=1}^{n} \frac{\tau_{j}}{\Gamma\left(\mathrm{q}-\lambda_{j}+1\right)}\left(\frac{\kappa_{j}^{\rho}-a^{\rho}}{\rho}\right)^{\mathrm{q}-\lambda_{j}}\|\vartheta-v\|\right) \\
& +\frac{2 \mathfrak{R}_{\mathrm{f}}}{1-\mathfrak{R}_{\mathrm{f}}} \frac{1}{\Gamma(\mathrm{q}+1)}\left(\frac{\sigma^{\rho}-\mathrm{a}^{\rho}}{\rho}\right)^{\mathrm{q}}\|\vartheta-v\| \\
\leqslant & \Delta\|\vartheta-v\| .
\end{aligned}
$$

Due to (3.1), the operator $\mathcal{S}$ is a contraction mapping on $\mathrm{C}(\mathrm{J})$.

Step (2): We need to show that such a fixed point $\vartheta \in C(J)$ is actually in $\mathrm{C}^{\gamma}(\mathrm{J})$. Let $\vartheta^{*}$ be a unique fixed point of operator $\mathcal{S}$ in $C(J)$, then

$$
\begin{aligned}
\vartheta^{*}(\sigma)= & \frac{\left(\frac{\sigma^{\rho}-a^{\rho}}{\rho}\right)^{\gamma-1}}{\Psi \Gamma(\gamma)}\left(B-\sum_{i=1}^{m} \theta_{i}{ }^{\rho_{\mathcal{J}}}{ }_{a^{+}}^{q+\psi_{i}} F_{\vartheta^{*}}(s)\left(\varpi_{i}\right)\right. \\
& \left.-\sum_{j=1}^{n} \tau_{j}{ }^{\rho} \mathcal{J}_{a^{+}}^{q-\lambda_{j}} F_{\vartheta^{*}}(s)\left(\kappa_{j}\right)\right)+{ }^{\rho_{\mathcal{J}}}{ }_{a^{+}}^{q} F_{\vartheta^{*}}(s)(\sigma) .
\end{aligned}
$$

Applying ${ }^{\rho} \mathrm{D}_{\mathrm{a}^{+}}^{\gamma}$ to both sides of the last equation, using Lemmas 2.4 and 2.5, we have

$$
{ }^{\rho} \mathrm{D}_{\mathrm{a}^{+}}^{\gamma} \vartheta^{*}(\sigma)={ }^{\rho} \mathrm{D}_{\mathrm{a}^{+}}^{\gamma}{ }^{\rho} \mathcal{J}_{\mathrm{a}^{+}}^{\mathrm{q}} \mathrm{F}_{\vartheta^{*}}(\mathrm{~s})(\sigma)={ }^{\rho} \mathrm{D}_{\mathrm{a}^{+}}^{\beta(1-\mathrm{q})} \mathrm{F}_{\vartheta^{*}}(\mathrm{~s})(\sigma),
$$

Since $\gamma \geqslant q$, by $\left(\mathrm{H}_{1}\right)$, we have $D_{a^{+}}^{\beta(1-\alpha)} \mathrm{F}_{\vartheta^{*}}(\mathrm{~s})(\sigma) \in \mathrm{C}(J)$, and hence ${ }^{\rho} \mathrm{D}_{\mathrm{a}^{+}}^{\gamma} \vartheta^{*}(\sigma) \in$ $\mathrm{C}(\mathrm{J})$. It follows from definition of $\mathrm{C}^{\gamma}(\mathrm{J})$ that $\vartheta^{*} \in \mathrm{C}^{\gamma}(\mathrm{J})$. As a consequence of the above steps, we conclude that the problem (1.1) has a unique solution in $\mathrm{C}^{\gamma}(\mathrm{J})$.

Theorem 3.3. Assume that $\left(H_{1}\right)$ holds. If

$$
\begin{aligned}
\Delta_{2}= & \frac{2 \Re_{f}}{\left(1-\mathfrak{R}_{f}\right) \Psi \Gamma(\gamma)}\left(|\mathrm{B}|+\sum_{i=1}^{m} \frac{\theta_{i}}{\Gamma\left(\mathrm{q}+\psi_{i}+1\right)}\left(\frac{\varpi_{i}^{\rho}-a^{\rho}}{\rho}\right)^{q+\psi_{i}}\right. \\
& \left.+\frac{2 \mathfrak{R}_{f}}{\left(1-\mathfrak{R}_{f}\right)} \sum_{j=1}^{n} \frac{\tau_{j}}{\Gamma\left(q-\lambda_{j}+1\right)}\left(\frac{\kappa_{j}^{\rho}-a^{\rho}}{\rho}\right)^{q-\lambda_{j}}\right)<1
\end{aligned}
$$

then the problem (1.1) has at least one solution on $\mathrm{J}$.

Proof. Consider a closed ball set $\mathrm{Q}_{\mathrm{r}}$ defined as

$$
\mathrm{Q}_{\mathrm{r}}=\{\vartheta \in \mathrm{C}(\mathrm{J}):\|\vartheta\| \leqslant \mathrm{r}\},
$$

with $r \geqslant \frac{\Delta_{1}}{1-\Delta_{2}}$, where

$$
\Delta_{1}=\frac{1}{\Psi \Gamma(\gamma)}\left(\sum_{i=1}^{m} \frac{\widehat{f}_{i}}{\Gamma\left(q+\psi_{i}+1\right)}\left(\frac{\varpi_{i}^{\rho}-a^{\rho}}{\rho}\right)^{q+\psi_{i}}+\sum_{j=1}^{n} \frac{\widehat{f} \tau_{j}}{\Gamma\left(q-\lambda_{j}+1\right)}\left(\frac{\kappa_{j}^{\rho}-a^{\rho}}{\rho}\right)^{q-\lambda_{j}}\right),
$$


and $\widehat{f}=\max _{\sigma \in \mathrm{J}}\left|\mathrm{F}_{0}(\sigma)\right|$, where $\mathrm{F}_{0}(\sigma)=\mathrm{f}(\sigma, 0,0,0)$. In order to prove existence solution by means of Krasnoselskii's (FPT), we split the operator $\mathcal{S}$ into tow operators $\mathcal{S}_{1}, \mathcal{S}_{2}$ such that $\mathcal{S}=\mathcal{S}_{1}+\mathcal{S}_{2}$, where

$$
\mathcal{S}_{1} \vartheta(\sigma):=\rho_{\mathcal{J}_{a^{+}}}^{q} F_{\vartheta}(s)(\sigma),
$$

and

$$
\begin{aligned}
\mathcal{S}_{2} \vartheta(\sigma)= & \frac{\left(\frac{\sigma^{\rho}-a^{\rho}}{\rho}\right)^{\gamma-1}}{\Psi \Gamma(\gamma)}\left(B-\sum_{i=1}^{m} \theta_{i} \rho_{\mathcal{J}}{ }_{a^{+}}^{q+\psi_{i}} F_{\vartheta}(s)\left(\varpi_{i}\right)\right. \\
& \left.-\sum_{j=1}^{n} \tau_{j} \rho_{\mathcal{J}_{a^{+}}^{q-}}^{q-\lambda_{j}} F_{\vartheta}(s)\left(\kappa_{j}\right)\right) .
\end{aligned}
$$

Step (I): $\mathcal{S}_{1} \vartheta(\sigma)+\mathcal{S}_{2} \vartheta(\sigma) \in \mathrm{Q}_{\mathrm{r}}$. For the operator $\mathcal{S}_{1}$ any $\vartheta \in \mathrm{Q}_{\mathrm{r}}, \sigma \in \mathrm{J}$, we have

$$
\begin{aligned}
& \left\|\mathcal{S}_{1} \vartheta\right\|=\max _{\sigma \in J}\left|\mathcal{S}_{1} \vartheta(\sigma)\right| \\
= & \left|\rho_{\mathcal{J}_{a^{+}}} F_{\vartheta}(s)(\sigma)\right| \\
\leqslant & \left.\rho_{\mathcal{J}_{a^{+}}^{q}}^{q}\left|F_{\vartheta}(s)-F_{0}(s)\right|+\left|F_{0}(s)\right|\right\}(\sigma) \\
\leqslant & \frac{2 \Re_{f}}{\left(1-\Re_{f}\right)} \frac{1}{\Gamma(q+1)}\left(\frac{\sigma^{\rho}-a^{\rho}}{\rho}\right)^{q}\|\vartheta\|+\frac{\widehat{f}}{\Gamma(q+1)}\left(\frac{\sigma^{\rho}-a^{\rho}}{\rho}\right)^{q} \\
\leqslant & \frac{2 \Re_{f}}{\left(1-\Re_{f}\right)} \frac{1}{\Gamma(q+1)}\left(\frac{T^{\rho}-a^{\rho}}{\rho}\right)^{q} r+\frac{\widehat{f}}{\Gamma(q+1)}\left(\frac{T^{\rho}-a^{\rho}}{\rho}\right)^{q} .
\end{aligned}
$$

For the operator $\mathcal{S}_{2}$ any $\vartheta \in \mathrm{Q}_{\mathrm{r}}, \sigma \in \mathrm{J}$, we have

$$
\begin{aligned}
& \left\|\mathcal{S}_{2} \vartheta\right\|=\max _{\sigma \in \mathrm{J}}\left|\mathcal{S}_{2} \vartheta(\sigma)\right| \\
\leqslant & \frac{1}{\Psi \Gamma(\gamma)}\left(|\mathrm{B}|+\sum_{i=1}^{m} \theta_{i} \rho_{\mathcal{J}_{a^{+}}^{q+\psi_{i}}}\left(\left|F_{\vartheta}(s)-F_{0}(s)+F_{0}(s)\right|\right)\left(\varpi_{i}\right)\right. \\
& \left.+\sum_{j=1}^{n} \tau_{j} \rho_{\mathcal{J}_{a^{+}}^{q-\lambda_{j}}}\left(\left|F_{\vartheta}(s)-F_{0}(s)+F_{0}(s)\right|\right)\left(\kappa_{j}\right)\right) \\
\leqslant & \frac{1}{\Psi \Gamma(\gamma)}\left(|B|+\sum_{i=1}^{m} \theta_{i} \rho_{\mathcal{J}_{a^{+}}^{q+}}^{q+\psi_{i}}\left\{\left|F_{\vartheta}(s)-F_{0}(s)\right|+\left|F_{0}(s)\right|\right\}\left(\varpi_{i}\right)\right. \\
& \left.+\sum_{j=1}^{n} \tau_{j} \rho_{\mathcal{J}_{a^{+}}^{q-\lambda_{j}}}^{q}\left\{\left|F_{\vartheta}(s)-F_{0}(s)\right|+\left|F_{0}(s)\right|\right\}\left(\kappa_{j}\right)\right)
\end{aligned}
$$




$$
\begin{aligned}
\leqslant & \frac{1}{\Psi \Gamma(\gamma)}\left(|B|+\frac{2 \Re_{f}}{1-\Re_{f}} \sum_{i=1}^{m} \frac{\theta_{i}}{\Gamma\left(q+\psi_{i}+1\right)}\left(\frac{\varpi_{i}^{\rho}-a^{\rho}}{\rho}\right)^{q+\psi_{i}}\right. \\
& \left.+\frac{2 \Re_{f}}{1-\Re_{f}} \sum_{j=1}^{n} \frac{\tau_{j} \Gamma(\gamma)}{\Gamma\left(q-\lambda_{j}+\gamma\right)}\left(\frac{\kappa_{j}^{\rho}-a^{\rho}}{\rho}\right)^{q-\lambda_{j}}\right) r \\
& +\frac{1}{\Psi \Gamma(\gamma)}\left(\sum_{i=1}^{m} \frac{\widehat{f} \theta_{i}}{\Gamma\left(q+\psi_{i}+1\right)}\left(\frac{\varpi_{i}^{\rho}-a^{\rho}}{\rho}\right)^{q+\psi_{i}}\right. \\
& \left.+\sum_{j=1}^{n} \frac{\widehat{f} \tau_{j}}{\Gamma\left(q-\lambda_{j}+1\right)}\left(\frac{\kappa_{j}^{\rho}-a^{\rho}}{\rho}\right)^{q-\lambda_{j}}\right)
\end{aligned}
$$

In the light of (3.3) and (3.4), we have

$$
\begin{aligned}
& \left\|\mathcal{S}_{1} \vartheta+\mathcal{S}_{2} v\right\| \\
& \leqslant \max \left\{\left\|\mathcal{S}_{1} \vartheta\right\|,\left\|\mathcal{S}_{2} v\right\|\right\} \\
& \leqslant \frac{1}{\Psi \Gamma(\gamma)}\left(|\mathrm{B}|+\frac{2 \mathfrak{R}_{\mathrm{f}}}{1-\mathfrak{R}_{\mathrm{f}}} \sum_{i=1}^{m} \frac{\theta_{i}}{\Gamma\left(\mathrm{q}+\psi_{i}+1\right)}\left(\frac{\varpi_{i}^{\rho}-\mathrm{a}^{\rho}}{\rho}\right)^{\mathrm{q}+\psi_{i}}\right. \\
& \left.+\frac{2 \Re_{f}}{1-\Re_{f}} \sum_{j=1}^{n} \frac{\tau_{j}}{\Gamma\left(q-\lambda_{j}+1\right)}\left(\frac{\kappa_{j}^{\rho}-a^{\rho}}{\rho}\right)^{q-\lambda_{j}}\right) r \\
& +\frac{1}{\Psi \Gamma(\gamma)}\left(\sum_{i=1}^{m} \frac{\widehat{f}_{i}}{\Gamma\left(q+\psi_{i}+1\right)}\left(\frac{\varpi_{i}^{\rho}-a^{\rho}}{\rho}\right)^{q+\psi_{i}}\right. \\
& \left.+\sum_{j=1}^{n} \frac{\widehat{f_{\tau}}}{\Gamma\left(q-\lambda_{j}+1\right)}\left(\frac{\kappa_{j}^{\rho}-a^{\rho}}{\rho}\right)^{q-\lambda_{j}}\right) \\
& \leqslant \Delta_{2} r+\Delta_{1} \leqslant r \text {. }
\end{aligned}
$$

which infers that $\mathcal{S}_{1} \vartheta+\mathcal{S}_{2} \vartheta \in \mathrm{Q}_{\mathrm{r}}$

Step (II): $\mathcal{S}_{2}$ is a contraction mapping. By Theorem 3.1, we deduce that $\mathcal{S}$ is a contraction mapping on $\mathrm{C}(\mathrm{J})$ and hence $S_{2}$ is a contraction mapping too.

Step (III): We prove that the operator $\mathcal{S}_{1}$ is compact and continuous.

The continuity of $\mathcal{S}_{1}$ follows from the continuity of $f$. From (3.3), we have

$$
\left\|\mathcal{S}_{1} \vartheta\right\| \leqslant \frac{2 \mathfrak{R}_{\mathrm{f}}}{1-\mathfrak{R}_{\mathrm{f}}} \frac{1}{\Gamma(\mathrm{q}+1)}\left(\frac{\mathrm{T}^{\rho}-\mathrm{a}^{\rho}}{\rho}\right)^{\mathrm{q}} \mathrm{r}+\frac{\widehat{f}}{\Gamma(\mathrm{q}+1)}\left(\frac{\mathrm{T}^{\rho}-\mathrm{a}^{\rho}}{\rho}\right)^{\mathrm{q}} .
$$

This means $\mathcal{S}_{1}$ is uniformly bounded. Now we prove the equicontinuous of operator $\mathcal{S}_{1}$. 
For any $\sigma_{1}, \sigma_{2} \in \mathrm{J}, \sigma_{1}<\sigma_{2}, \vartheta \in \mathrm{Q}_{\mathrm{r}}$, we get

$$
\begin{aligned}
& \left|\mathcal{S}_{1} \vartheta\left(\sigma_{2}\right)-\mathcal{S}_{1} \vartheta\left(\sigma_{1}\right)\right| \\
= & \mid \frac{1}{\Gamma(\mathrm{q})} \int_{a}^{\sigma_{2}}\left(\frac{\sigma_{2}^{\rho}-s^{\rho}}{\rho}\right)^{\mathrm{q}-1} s^{\rho-1} \mathrm{~F}_{\vartheta}(s) \mathrm{d} s \\
& -\frac{1}{\Gamma(\mathrm{q})} \int_{a}^{\sigma_{1}}\left(\frac{\sigma_{1}^{\rho}-s^{\rho}}{\rho}\right)^{\mathrm{q}-1} s^{\rho-1} \mathrm{~F}_{\vartheta}(s) \mathrm{d} s \mid \\
\leqslant & \frac{\left\|\mathrm{F}_{\vartheta}(\cdot)\right\|}{\Gamma(\mathrm{q})}\left|\int_{a}^{\sigma_{1}} s^{\rho-1}\left(\left(\frac{\sigma_{2}^{\rho}-s^{\rho}}{\rho}\right)^{\mathrm{q}-1}-\left(\frac{\sigma_{1}^{\rho}-s^{\rho}}{\rho}\right)^{\mathrm{q}-1}\right) \mathrm{d} s\right| \\
& +\frac{\left\|\mathrm{F}_{\vartheta}(\cdot)\right\|}{\Gamma(\mathrm{q})}\left|\int_{\sigma_{1}}^{\sigma_{2}} s^{\rho-1}\left(\frac{\sigma_{2}^{\rho}-s^{\rho}}{\rho}\right)^{\mathrm{q}-1} \mathrm{~d} s\right| \\
& \rightarrow 0 \text { as } \sigma_{2} \rightarrow \sigma_{1} .
\end{aligned}
$$

Thus $\mathcal{S}_{1}$ is equicontinuous. Hence, it follows from the Arzelà-Ascoli theorem that the operator $\mathcal{S}_{1}$ is compact on $Q_{r}$. According to Theorem 2.7, we conclude that $\mathcal{S}$ has at least a fixed point $\vartheta^{*} \in \mathrm{C}(\mathrm{J})$ and by the same technique of Theorem 3.2, one can show that the problem (1.1) has at least one solution.

\section{4. $\delta$-approximate solution}

Definition 4.1. A function $\vartheta \in \mathrm{C}(\mathrm{J})$ satisfying the following inequality

$$
\left\|\rho D_{a^{+}}^{q, \beta} \vartheta(\sigma)-F_{\vartheta}(\sigma)\right\| \leqslant \delta, \sigma \in J,
$$

and

$$
\sum_{i=1}^{m} \theta_{i} \rho \mathcal{J}_{a^{+}}^{\psi_{i}} \vartheta\left(\varpi_{i}\right)=B^{*}-\sum_{j=1}^{n} \tau_{j}^{\rho} D_{a^{+}}^{\lambda_{j}, \beta} \vartheta\left(\kappa_{j}\right), B^{*} \in \mathbb{R} .,
$$

is called $\delta$-approximate solutions of Hilfer-Katugampola fractional differential (1.1).

Theorem 4.2. Let $\mathrm{F}: \mathrm{J} \times \mathbb{R} \times \mathbb{R} \times \mathbb{R} \rightarrow \mathbb{R}$ be a continuous function satisfies the condition $\left(H_{2}\right)$ for each $\sigma \in \mathrm{J}$ and let $\vartheta_{1}, \vartheta_{2} \in \mathrm{C}(\mathrm{J})$ be a $\delta$-approximation solutions of the following Hilfer-Katugampola fractional differential equations

$$
\begin{gathered}
\rho \mathrm{D}_{\mathrm{a}^{+}}^{\mathrm{q}, \beta} \vartheta_{1}(\sigma)=\mathrm{F}_{\vartheta_{1}}(\sigma), \\
\sum_{i=1}^{m} \theta_{i} \rho \mathcal{J}_{\mathrm{a}^{+}}^{\psi_{i}} \vartheta_{1}\left(\Phi_{i}\right)=B_{1}^{*}-\sum_{j=1}^{n} \tau_{j} \rho D_{a^{+}}^{\lambda_{j}, \beta} \vartheta_{1}\left(\kappa_{j}\right),
\end{gathered}
$$

and

$$
\begin{gathered}
\rho \mathrm{D}_{\mathrm{a}^{+}}^{\mathrm{q}, \beta} \vartheta_{2}(\sigma)=\mathrm{F}_{\vartheta_{2}}(\sigma), \\
\sum_{i=1}^{m} \theta_{i} \rho \mathcal{J}_{a^{+}}^{\psi_{i}} \vartheta_{2}\left(\varpi_{i}\right)=B_{2}^{*}-\sum_{j=1}^{n} \tau_{j} \rho D_{a^{+}}^{\lambda_{j}, \beta} \vartheta_{2}\left(k_{j}\right),
\end{gathered}
$$

Then

$$
\left\|\vartheta_{1}-\vartheta_{2}\right\| \leqslant \frac{1}{1-\Delta}\left(\frac{\delta_{1}+\delta_{2}}{\Gamma(q+1)}\left(\frac{T^{\rho}-a^{\rho}}{\rho}\right)^{q}+\frac{\left|B_{1}^{*}-B_{2}^{*}\right|}{\Psi \Gamma(\gamma)}\right) .
$$


Proof. Let $\vartheta_{1}, \vartheta_{2} \in \mathrm{C}(\mathrm{J})$ be an $\delta$-approximation solutions of the problem (1.1). Then, we have

$$
\left\{\begin{array}{l}
\| \rho D_{a^{+}}^{q, \beta} \vartheta_{1}(\sigma)-F_{\vartheta_{1}}(\sigma) \\
\rho D_{a^{+}}^{q, \beta} \vartheta_{2}(\sigma)-F_{\vartheta_{2}}(\sigma)
\end{array} \| \leqslant \delta_{1}\right.
$$

and

$$
\left\{\begin{array}{l}
\sum_{i=1}^{m} \theta_{i} \rho \mathcal{J}_{a^{+}}^{\psi_{i}} \vartheta_{1}\left(\varpi_{i}\right)=B_{1}^{*}-\sum_{j=1}^{n} \tau_{j}{ }^{\rho} D_{a_{a^{+}}}^{\lambda_{j}, \beta} \vartheta_{1}\left(\kappa_{j}\right) \\
\sum_{i=1}^{m} \theta_{i} \rho \mathcal{J}_{a^{+}}^{\psi_{i}} \vartheta_{2}\left(\varpi_{i}\right)=B_{2}^{*}-\sum_{j=1}^{n} \tau_{j} \rho D_{a^{+}}^{\lambda_{j}, \beta} \vartheta_{2}\left(\kappa_{j}\right) .
\end{array}\right.
$$

Applying $\rho \mathcal{J}_{\mathrm{a}^{+}}^{\mathrm{q}}$ on both sides of the above inequality, and using lemma 2.6, we get

$$
\begin{aligned}
& \mid \vartheta_{1}(\sigma)-\frac{\left(\frac{\sigma^{\rho}-a^{\rho}}{\rho}\right)^{\gamma-1}}{\Psi \Gamma(\gamma)} B_{1}^{*}+\frac{\left(\frac{\sigma^{\rho}-a^{\rho}}{\rho}\right)^{\gamma-1}}{\Psi \Gamma(\gamma)} \sum_{i=1}^{m} \theta_{i} \rho_{\mathcal{J}_{a^{+}}^{q+} \psi_{i}} F_{\vartheta_{1}}(s)\left(\varpi_{i}\right) \\
& \quad+\frac{\left(\frac{\sigma^{\rho}-a^{\rho}}{\rho}\right)^{\gamma-1}}{\Psi \Gamma(\gamma)} \sum_{j=1}^{n} \tau_{j} \rho_{\mathcal{J}_{a^{+}}^{q-} \lambda_{j}} F_{\vartheta_{1}}(s)\left(\kappa_{j}\right)-\rho_{\mathcal{J}_{a^{+}}^{q}} F_{\vartheta_{1}}(s)(\sigma) \mid \\
& \leqslant \frac{\delta_{1}}{\Gamma(q+1)}\left(\frac{\sigma^{\rho}-a^{\rho}}{\rho}\right)^{q}
\end{aligned}
$$

and

$$
\begin{aligned}
& \mid \vartheta_{2}(\sigma)-\frac{\left(\frac{\sigma^{\rho}-a^{\rho}}{\rho}\right)^{\gamma-1}}{\Psi \Gamma(\gamma)} B_{1}^{*}+\frac{\left(\frac{\sigma^{\rho}-a^{\rho}}{\rho}\right)^{\gamma-1}}{\Psi \Gamma(\gamma)} \sum_{i=1}^{m} \theta_{i} \rho_{\mathcal{J}_{a^{+}}^{q+}}^{q+\psi_{i}} F_{\vartheta_{2}}(s)\left(\varpi_{i}\right) \\
& \quad+\frac{\left(\frac{\sigma^{\rho}-a^{\rho}}{\rho}\right)^{\gamma-1}}{\Psi \Gamma(\gamma)} \sum_{j=1}^{n} \tau_{j} \rho_{\mathcal{J}_{a^{+}}^{q-\lambda}} F_{\vartheta_{2}}(s)\left(\kappa_{j}\right)-\rho_{\mathcal{J}_{a^{+}}^{q}} F_{\vartheta_{2}}(s)(\sigma) \mid \\
& \leqslant \frac{\delta_{2}}{\Gamma(q+1)}\left(\frac{\sigma^{\rho}-a^{\rho}}{\rho}\right)^{q}
\end{aligned}
$$

Using the fact $|x|-|y| \leqslant|x-y| \leqslant|x|+|y|$ in the above inequality, we get

$$
\begin{aligned}
& \left|\left(\vartheta_{1}(\sigma)-\vartheta_{2}(\sigma)\right)\right| \\
& \leqslant \frac{\delta_{1}+\delta_{2}}{\Gamma(\mathrm{q}+1)}\left(\frac{\sigma^{\rho}-\mathrm{a}^{\rho}}{\rho}\right)^{\mathrm{q}}+\left|\left(\mathrm{B}_{1}^{*}-\mathrm{B}_{2}^{*}\right) \frac{1}{\Psi \Gamma(\gamma)}\right| \\
& +\frac{1}{\Psi \Gamma(\gamma)} \sum_{i=1}^{m} \theta_{i}{ }^{\rho} \mathcal{J}_{a^{+}}^{q+\psi_{i}}\left|F_{\vartheta_{1}}(s)-F_{\vartheta_{1}}(s)\right|\left(\varpi_{i}\right) \\
& \frac{1}{\Psi \Gamma(\gamma)} \sum_{j=1}^{n} \tau_{j} \rho_{\mathcal{J}_{a^{+}}^{q-\lambda_{j}}}^{q} F_{\vartheta_{1}}(s)-F_{\vartheta_{1}}(s) \mid\left(\kappa_{j}\right) \\
& +{ }^{\rho} \mathcal{J}_{a^{+}}^{q}\left|F_{\vartheta_{1}}(s)-F_{\vartheta_{1}}(s)\right|(\sigma)
\end{aligned}
$$




$$
\begin{aligned}
\leqslant & \frac{\delta_{1}+\delta_{2}}{\Gamma(\mathrm{q}+1)}\left(\frac{T^{\rho}-a^{\rho}}{\rho}\right)^{\mathrm{q}}+\left|\left(B_{1}^{*}-B_{2}^{*}\right) \frac{1}{\Psi \Gamma(\gamma)}\right| \\
& +\frac{2 \mathfrak{R}_{\mathrm{f}}}{1-\mathfrak{R}_{\mathrm{f}}} \frac{1}{\Psi \Gamma(\gamma)} \sum_{i=1}^{\mathrm{m}} \frac{\theta_{i}}{\Gamma\left(\mathrm{q}+\psi_{i}+1\right)}\left(\frac{\varpi_{i}^{\rho}-\mathrm{a}^{\rho}}{\rho}\right)^{\mathrm{q}+\psi_{i}}\left\|\vartheta_{1}-\vartheta_{2}\right\| \\
& +\frac{2 \mathfrak{R}_{\mathrm{f}}}{1-\mathfrak{R}_{\mathrm{f}}} \frac{1}{\Psi \Gamma(\gamma)} \sum_{j=1}^{n} \frac{\tau_{j}}{\Gamma\left(\mathrm{q}-\lambda_{j}+1\right)}\left(\frac{\kappa_{j}^{\rho}-a^{\rho}}{\rho}\right)^{\mathrm{q}-\lambda_{j}+\gamma-1}\left\|\vartheta_{1}-\vartheta_{2}\right\| \\
& +\frac{2 \mathfrak{R}_{\mathrm{f}}}{1-\mathfrak{R}_{\mathrm{f}}} \frac{1}{\Gamma(\mathrm{q}+1)}\left(\frac{T^{\rho}-\mathrm{a}^{\rho}}{\rho}\right)^{\mathrm{q}}\left\|\vartheta_{1}-\vartheta_{2}\right\| \\
\leqslant & \frac{\delta_{1}+\delta_{2}}{\Gamma(\mathrm{q}+1)}\left(\frac{T^{\rho}-\mathrm{a}^{\rho}}{\rho}\right)^{\mathrm{q}}+\frac{\left|\mathrm{B}_{1}^{*}-\mathrm{B}_{2}^{*}\right|}{\Psi \Gamma(\gamma)}+\Delta\left\|\vartheta_{1}-\vartheta_{2}\right\| .
\end{aligned}
$$

In consequence, we have

$$
\begin{aligned}
& \left\|\vartheta_{1}-\vartheta_{2}\right\| \\
\leqslant & \frac{1}{1-\Delta}\left(\frac{\delta_{1}+\delta_{2}}{\Gamma(\mathrm{q}+1)}\left(\frac{\mathrm{T}^{\rho}-\mathrm{a}^{\rho}}{\rho}\right)^{\mathrm{q}}+\frac{\left|\mathrm{B}_{1}^{*}-\mathrm{B}_{2}^{*}\right|}{\Psi \Gamma(\gamma)}\right) .
\end{aligned}
$$

Remark 4.3. If $\delta_{1}=\delta_{2}=0$ in the inequality (4.3), then $\vartheta_{1}, \vartheta_{2}$ are solutions of the problem (1.1) and the inequality (4.4) reduces to

$$
\left\|\vartheta_{1}-\vartheta_{2}\right\| \leqslant \frac{1}{1-\Delta}\left(\frac{\left|B_{1}^{*}-B_{2}^{*}\right|}{\Psi \Gamma(\gamma)}\right) .
$$

which provides the continuous dependence of the problem (1.1). Also if $B_{1}^{*}=B_{2}^{*}$, we have $\left\|\vartheta_{1}-\vartheta_{2}\right\|=0$, which provides the uniqueness of a solution of problem (1.1).

\section{Examples}

Example 5.1. Consider the following problem

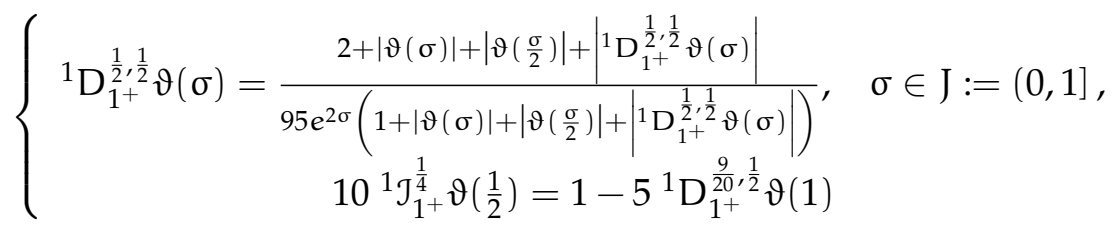

Here $\mathrm{q}=\frac{1}{2}, \beta=\frac{1}{2}, \gamma=\mathrm{q}+\beta-\mathrm{q} \beta=\frac{3}{4}, \mu=\frac{1}{2}, \mathrm{~m}=\mathrm{n}=1, \theta_{1}=10, \tau_{1}=5, \varpi_{1}=\frac{1}{2}$, $\mathrm{\kappa}_{1}=1, \mathrm{~B}=1,(\mathrm{a}, \mathrm{T}]=(0,1], \rho=1, \psi_{1}=\frac{1}{4}, \lambda_{1}=\frac{9}{20}$ and

$$
f\left(\sigma, \vartheta(\sigma), \vartheta\left(\frac{\sigma}{2}\right),{ }^{\rho} D_{a^{+}}^{q, \beta} \vartheta(\sigma)\right)=\frac{2+|\vartheta(\sigma)|+\left|\vartheta\left(\frac{\sigma}{2}\right)\right|+\left|{ }^{1} D_{1^{+}}^{\frac{1}{2}, \frac{1}{2}} \vartheta(\sigma)\right|}{95 e^{2 \sigma}\left(1+|\vartheta(\sigma)|+\left|\vartheta\left(\frac{\sigma}{2}\right)\right|+\left|{ }^{1} D_{1^{+}}^{\frac{1}{2}, \frac{1}{2}} \vartheta(\sigma)\right|\right)} .
$$


Thus, $f\left(\sigma, \vartheta(\sigma), \vartheta(\mu(\sigma)),{ }^{\rho} D_{a^{+}}^{q, \beta} \vartheta(\sigma)\right)$ is continuous and $f\left(\cdot, \vartheta(\cdot), \vartheta(\lambda \cdot),{ }^{\rho} D_{a^{+}}^{q, \beta} \vartheta(\cdot)\right) \in C(J)$ for all $\vartheta \in \mathrm{C}(\mathrm{J})$. Let $\sigma \in(0,1]$ and $\vartheta, \bar{\vartheta} \in \mathbb{R}$. Then

$$
\begin{aligned}
& \left|f\left(\sigma, \vartheta(\sigma), \vartheta\left(\frac{\sigma}{2}\right),{ }^{1} D_{1^{+}}^{\frac{1}{2}, \frac{1}{2}} \vartheta(\sigma)\right)-f\left(\sigma, \bar{\vartheta}(\sigma), \bar{\vartheta}\left(\frac{\sigma}{2}\right),{ }^{1} D_{1^{+}}^{\frac{1}{2}, \frac{1}{2}} \bar{\vartheta}(\sigma)\right)\right| \\
\leqslant & \frac{1}{95}\left(|\vartheta(\sigma)-\bar{\vartheta}(\sigma)|+\left|\vartheta\left(\frac{\sigma}{2}\right)-\bar{\vartheta}\left(\frac{\sigma}{2}\right)\right|+\left|{ }^{1} D_{1^{+}}^{\frac{1}{2}, \frac{1}{2}} \vartheta(\sigma)-{ }^{1} D_{1^{+}}^{\frac{1}{2}, \frac{1}{2}} \bar{\vartheta}(\sigma)\right|\right) .
\end{aligned}
$$

Thus the condition $\left(\mathrm{H}_{1}\right)$ is satisfied with $\mathfrak{R}_{\mathrm{f}}=\frac{1}{95}$. Furthermore, by simple calculation, we get $\Psi \simeq 11.67$ and $\Delta \simeq 0.049<1$. Then all the assumptions in Theorem 3.2 are satisfied. Hence, the problem $(5.1)$ has a unique solution in $C^{\frac{1}{2}}([0,1])$. Also, $\Delta_{2} \simeq 0.019<1$. It follows from Theorem 3.3 the problem (5.1) has at least one solution.

Example 5.2. Consider the following problem

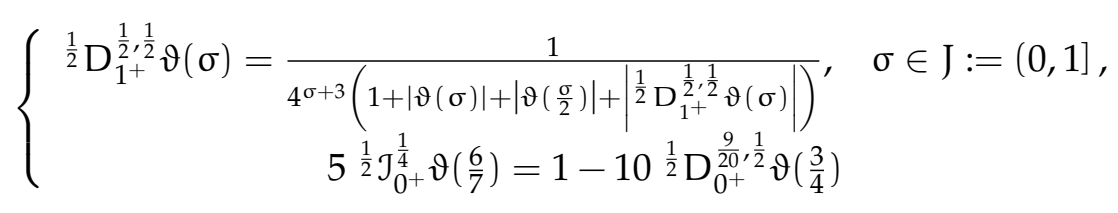

Here $q=\frac{1}{2}, \beta=\frac{1}{2}, \gamma=q+\beta-q \beta=\frac{3}{4}, m=n=1, \mu=\frac{1}{2}, \theta_{1}=5, \tau_{1}=10, \varpi_{1}=\frac{6}{7}$, $\kappa_{1}=\frac{3}{4}, \mathrm{~B}=1,(\mathrm{a}, \mathrm{T}]=(0,1], \rho=\frac{1}{2}, \psi_{1}=\frac{1}{4}, \lambda_{1}=\frac{9}{20}$ and

$$
\left.f\left(\sigma, \vartheta(\sigma), \vartheta\left(\frac{\sigma}{2}\right)\right)^{\frac{1}{2}} D_{1^{+}}^{\frac{1}{2}, \frac{1}{2}} \vartheta(\sigma)\right)=\frac{1}{4^{\sigma+3}\left(1+|\vartheta(\sigma)|+\left|\vartheta\left(\frac{\sigma}{2}\right)\right|+\left|\frac{1}{2} D_{1^{+}}^{\frac{1}{2}, \frac{1}{2}} \vartheta(\sigma)\right|\right)}
$$

. Thus, $f\left(\sigma, \vartheta(\sigma), \vartheta(\mu(\sigma)),{ }^{\rho} D_{a^{+}}^{q, \beta} \vartheta(\sigma)\right)$ is continuous and $f\left(\cdot, \vartheta(\cdot), \vartheta(\mu(\cdot)),{ }^{\rho} D_{a^{+}}^{q, \beta} \vartheta(\cdot)\right) \in C(J)$ for all $\vartheta \in C(J)$. Let $\sigma \in(0,1]$ and $\vartheta, \bar{\vartheta} \in \mathbb{R}$. Then

$$
\begin{aligned}
& \left|f\left(\sigma, \vartheta(\sigma), \vartheta\left(\frac{\sigma}{2}\right), \frac{1}{2} D_{1^{+}}^{\frac{1}{2}, \frac{1}{2}} \vartheta(\sigma)\right)-f\left(\sigma, \bar{\vartheta}(\sigma), \bar{\vartheta}\left(\frac{\sigma}{2}\right), \frac{1}{2} D_{1^{+}}^{\frac{1}{2}, \frac{1}{2}} \bar{\vartheta}(\sigma)\right)\right| \\
\leqslant & \frac{1}{64}\left(|\vartheta(\sigma)-\bar{\vartheta}(\sigma)|+\left|\vartheta\left(\frac{\sigma}{2}\right)-\bar{\vartheta}\left(\frac{\sigma}{2}\right)\right|+\left|\frac{1}{2} D_{1^{+}}^{\frac{1}{2}, \frac{1}{2}} \vartheta(\sigma)--^{\frac{1}{2}} D_{1^{+}}^{\frac{1}{2}, \frac{1}{2}} \bar{\vartheta}(\sigma)\right|\right) .
\end{aligned}
$$

Thus the condition $\left(\mathrm{H}_{1}\right)$ is satisfied with $\mathfrak{R}_{\mathrm{f}}=\frac{1}{64}$. Furthermore, by simple calculation, we get $\Psi \simeq 7.27$ and $\Delta \simeq 0.031<1$. Then all the assumptions in Theorem 3.2 are satisfied. Hence, the problem (5.1) has a unique solution in $\mathrm{C}^{\frac{1}{2}}([0,1])$. Also, $\Delta_{2}<1$. It follows from Theorem 3.3 the problem (5.1) has at least one solution.

\section{Conclusion}

In this work, the existence and uniqueness of the nonlocal boundary value problem for differential equation with Hilfer-Katugampola fractional derivative were discussed. Krasnoselskii fixed point theorem and Banach contraction principle are utilized to obtain our results. The dependence of solutions and uniqueness have been obtained by means of $\delta$ -approximated solution. In conclusion, Hilfer-Katugampola fractional derivative can be 
used as a powerful tool for studying the dynamical behavior of many real-world problems. The acquired results which are obtained in the current paper are more general and cover many of the parallel problems that contain special cases of function because our proposed problem contains a global fractional derivative that integrates many classic fractional derivatives.

\section{References}

[1] Kilbas AA, Srivastava HM and Trujillo JJ (2006). "Theory and Applications of Fractional Differential Equations". North-Holland Mathematics Studies, Elsevier, Amsterdam, 207.

[2] Samko SG, Kilbas AA and Marichev OI (1987). "Fractional Integrals and Derivatives,": Theory and Applications, Gordon and Breach, Yverdon.

[3] Podlubny I (1990). "Fractional Differential Equations,", Math. Sci. Eng., 198, Elsevier, Amsterdam, 1999.

[4] Hilfer R (1999). "Applications of Fractional Calculus in Physics". World scientic, Singapore.

[5] Hilfer R (2008). "Threefold Introduction to Fractional Derivatives". Anomalous Transport: Foundations and Applications; Wiley-VCH,Weinheim, Germany.17.

[6] Katugampola UA (2011). New approach to a generalized fractional integral. Appl. Math. Comput. 218: 860-865.

[7] Baleanu D, Diethelm K, Scalas E and Trujillo J.J (2012). Fractional Calculus Models and Numerical Methods; World Scientific Publishing: New York, NY, USA, .

[8] Baleanu D, Güvenç ZB and Machado JAT (2010). New Trends in Nanotechnology and Fractional Calculus Applications; Springer: New York, NY, USA.

[9] Gambo YY, Jarad F, Baleanu D and Abdeljawad T (2014). On Caputo modification of the Hadamard fractional derivatives. Adv. Differ. Equ. 1: 1-12. https://doi.org/10.1186/1687-1847-2014-10

[10] Almalahi MA, Abdo MS and Panchal SK (2020). భ-Hilfer Fractional functional differential equation by Picard operator method. J. Nonlinear Dyn. 9(4): 685-702. https://doi.org/10.5890/JAND.2020.12.011

[11] Almalahi MA, Panchal SK (2021). E $E_{\alpha}$-Ulam-Hyers stability result for $\psi$-Hilfer Nonlocal Fractional Differential Equation. Disc. Nonlin. Complexity. 172: (2021).

[12] Almalahi MA, Abdo MS and Panchal SK (2020). Existence and Ulam-Hyers-Mittag-Leffler stability results of $\psi$-Hilfer nonlocal Cauchy problem. Rend. Circ. Mat. Palermo, II. Ser (2020). https://doi.org/10.1007/s12215-020-00484-8

[13] Almalahi, MA and Panchal SK (2020). Some existence and stability results for $\psi$-Hilfer fractional implicit differential equation with periodic conditions. J. Math. Anal. Model. 1(1): 1-19. https://doi.org/10.48185/jmam.v1i1.4

[14] Abdo MS (2020). Further results on the existence of solutions for generalized fractional quadratic functional integral equations. J. Math. Anal. Model. 1(1): 33-46. https://doi.org/10.48185/jmam.v1i1.2

[15] Wahash HA and Panchal SK. (2020). Positive solutions for generalized two-term fractional differential equations with integral boundary conditions. J. Math. Anal. Model. 1(1), 47-63. https://doi.org/10.48185/jmam.v1i1.35

[16] Wahash HA, Panchal SK (2020). Positive solutions for generalized Caputo fractional differential equations using lower and upper solutions method. J. Frac. Calc. Nonlinear Sys. 1(1): 1-12. https://doi.org/10.48185/jfcns.v1i1.78

[17] Redhwan S, Shaikh S (2020). Analysis of implicit type of a generalized fractional differential equations with nonlinear integral boundary conditions. J. Math. Anal. Model. 1(1), 64-76. https://doi.org/10.48185/jmam.v1i1.14

[18] Redhwan S, Shaikh SL (2021). Implicit fractional differential equation with nonlocal integral-multipoint boundary conditions in the frame of Hilfer fractional derivative. J. Math. Anal. Model. 2(1): 62-71. https://doi.org/10.48185/jmam.v2i1.176

[19] Ockendon JR and Tayler AB (1971). "The dynamics of a current collection system for an electric locomotive". Proc. R. Soc. Lond. Ser. A, Math. Phys. Sci. 322(1551): 447-468. http://doi.org/10.1098/rspa.1971.0078

[20] Balachandran K, Kiruthika S and Trujillo JJ (2013). Existence of solutions of nonlinear fractional pantograph equations. Acta Math. Sci. 33(3): 712-720. https://doi.org/10.1016/S0252-9602(13)60032-6 
[21] Vivek D, Kanagarajan K and Sivasundaram S (2017). Theory and analysis of nonlinear neutral pantograph equations via Hilfer fractional derivative. Nonlinear Stud. 24(3): 699-712

[22] Anguraj A, Vinodkumar A and Malar K (2016). Existence and stability results for random impulsive fractional pantograph equations. Filomat 30(14): 3839-3854. http://www.jstor.org/stable/24899471

[23] Elsayed E, Harikrishnan S and Kanagarajan K (2018). Analysis of nonlinear neutral pantograph differential equations with Hilfer fractional derivative. MathLAB 1: 231-240

[24] Harikrishnan S, Ibrahim R and Kanagarajan K (2018). Establishing the existence of Hilfer fractional pantograph equations with impulses. Fundam. J. Math. Appl. 1(1): 36-42. https://doi.org/10.33401/fujma.406651

[25] Ahmed I, Kumam P, Shah K., et al (2020). Stability results for implicit fractional pantograph differential equations via Hilfer fractional derivative with a nonlocal Riemann-Liouville fractional integral condition. Mathematics 8(1), 94. https://doi.org/10.3390/math8010094

[26] Abdo MS, Abdeljawad T, Shah K, Ali SM (2021). On nonlinear coupled evolution system with nonlocal subsidiary conditions under fractal-fractional order derivative. Math. Meth. Appl. Sci. 44(8): 6581-6600. https://doi.org/10.1002/mma.7210

[27] Abdo M.S, Abdeljawad T, Kucche K.D, Alqudah M.A, Ali, S.M and Jeelani M.B. (2021). On nonlinear pantograph fractional differential equations with Atangana-Baleanu-Caputo derivative. Adv. Diff. Equ, 2021(1), 1-17. https://doi.org/10.1186/s13662-021-03229-8

[28] Asawasamrit S, Woraphak N, Sotiris K, Ntouyas and Jessada T (2019). Existence and stability analysis for fractional differential equations with mixed nonlocal conditions. Mathematics. 7: 117. https://doi.org/10.3390/math7020117

[29] Almalahi MA and Panchal SK (2020). Existence and stability results of relaxation fractional differential equations with Hilfer-Katugampola fractional derivative. Advances in the Theory of Nonlinear Analysis and its Application. 4: 299-315. https://doi.org/10.31197/atnaa.686693

[30] Katugampola UN (2011). New approach to a genaralized fractional integral. Appl. Math.Comput., 3(218): 860-865. https://doi.org/10.1016/j.amc.2011.03.062

[31] Oliveira DS and Capelas de oliveira E (2017). Hilfer-Katugampola fractional derivative. Comp Appl Math. 37: 3672-3690. https://doi.org/10.1007/s40314-017-0536-8

[32] Burton TA (1998). "A fixed-point theorem of Krasnoselskii" App. Math. Letters. 11(1): 85-88. 